\title{
Two Giant Planets Orbiting the K Giant Star $\eta$ Cet
}

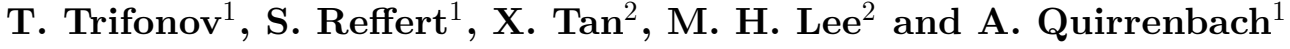 \\ ${ }^{1}$ ZAH-Landessternwarte, Königstuhl 12, 69117 Heidelberg, Germany \\ ${ }^{2}$ Department of Earth Sciences, The University of Hong Kong, Pokfulam Road, Hong Kong
}

\begin{abstract}
We present evidence of a new planetary system around the K giant $\eta$ Cet (HIP 5364, HD 6805, HR 334), based on 124 high-precision optical and infrared radial velocity data, taken at Lick Observatory (Hamilton) and at VLT (CRIRES). The best dynamical fit to the data is consistent with two massive planets $\left(m_{1} \sin i \approx 2.6 M_{\text {Jup }}, m_{2} \sin i \approx 3.3 M_{\text {Jup }}\right)$ and with periods of $P_{1} \approx 407$ days, $P_{2} \approx 740$ days. To test the $\eta$ Cet system's stability we perform $\sim 10,000$ dynamical investigations with maximum time spans of $10^{8}$ years. We find that in case of moderate eccentricities, the planets can be effectively trapped in an anti-aligned stable 2:1 mean motion resonance (MMR), very close to the separatrix. A larger non-resonant stable region exists in low-eccentricity parameter space, although less probable than the 2:1 MMR region.
\end{abstract}

Keywords. techniques: radial velocities, methods: numerical, planetary systems

\section{Introduction}

More than 133 known multiple-planet systems are present in the literature up to June 2013; their number is constantly growing. Nevertheless, not many multiple planetary systems have been found around evolved giant stars so far. For more than a decade we have been studying 373 very bright ( $\mathrm{V} \leqslant 6 \mathrm{mag}$ ) $\mathrm{G}$ and $\mathrm{K}$ giants (Frink et al. 2001) using high precision Doppler spectroscopy in an attempt to discover planetary companions around intermediate-mass stars. In this study we report the detection of two Jovian planets orbiting the $\mathrm{K}$ giant $\eta$ Cet. This system is only the fourth known planetary system around a $\mathrm{G}$ and $\mathrm{K}$ giant stars and presents an important milestone for understanding planetary formation and evolution as a function of stellar mass, metallicity and age.

\section{Observations}

Between Jul. 2000 and Dec. 2011, 118 optical Doppler measurements for $\eta$ Cet have been obtained with the Hamilton Échelle Spectrograph at Lick Observatory $(R \approx 60,000$, $\left.\sigma=3-5 \mathrm{~ms}^{-1}\right)$. Starting Oct. 2011, six additional near-IR data points have been taken with CRIRES $\left(R \approx 100,000, \sigma=40 \mathrm{~ms}^{-1}\right)$. Assuming the mass of $\eta$ Cet to be $1.7 M_{\odot}$ (Reffert et al. submitted) the dynamical model suggests that the $\eta$ Cet system contains two strongly interacting giant planets. The orbital parameters and their estimated errors from the best dynamical fit are summarized in Table 1.

\section{Dynamical tests}

For the stability test we have used the Mercury N-body simulator (Chambers 1999). We took input orbital parameters from a grid of best fits for fixed $e_{1}$ and $e_{2}$ (Fig. $1 \mathrm{f}, \mathrm{g}$ ). Each grid pixel is an output orbital parameter set from the orbit fitting routine, with the stellar jitter (r.m.s. $=15.1 \mathrm{~ms}^{-} 1$ ) quadratically added into $\sigma_{R V}$. Using those grids as an input ( $\sim 10,000$ fits), we find two permitted stability regions for the $\eta$ Cet planetary system: either non-resonant in near-circular orbits, or with higher probability in a moderate $e_{2}$ regime, where the system appears to be in an anti-aligned 2:1 MMR. In this configuration the lowest order eccentricity-type resonant angle $\theta_{1}$ librates around $180^{\circ}$ and $\theta_{2}$ librates 

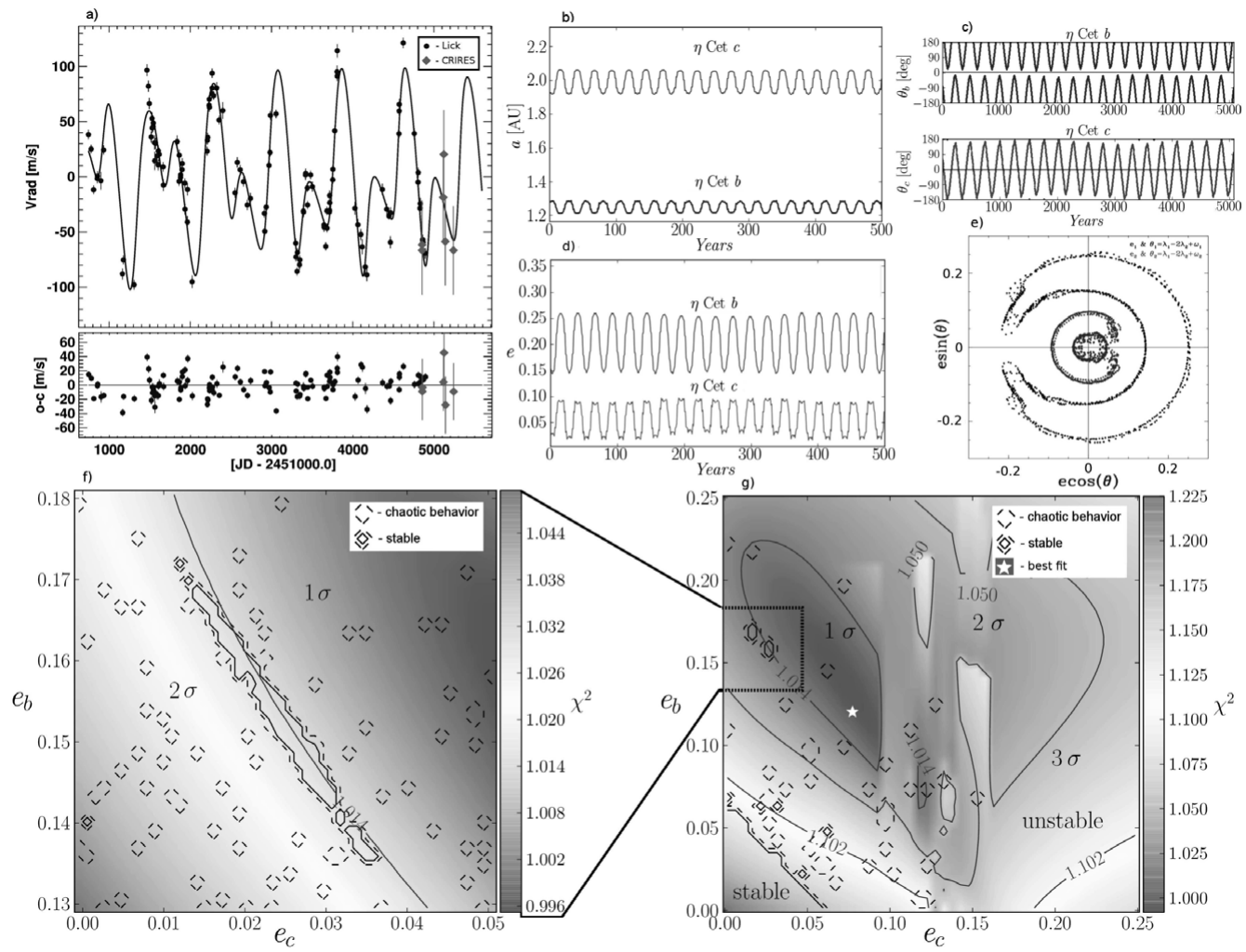

Figure 1. a) The best fit model to the data. b) Semi-major axis evolution: the planets exhibit strong perturbations, but are bound in stable orbits for at least $10^{8}$ yr. The mean period ratio is $2: 1$. d) Eccentricities are rapidly changing with the same phase. c) The resonance angles $\theta_{1}$ and $\theta_{2}$ librate with very large amplitudes of $\sim \pm 180^{\circ}$ - very close to the separatrix, however e) shows that the system is always in an anti-aligned planetary configuration. g) Illustrates the two stable regions in the $e_{1} / e_{2}$ plane, while f) shows the 2:1 MMR region with higher resolution.

Table 1. Best fit orbital parameters of the $\eta$ Cet planetary system.

\begin{tabular}{lccccc}
\hline \hline & $P[$ days $]$ & $m \sin i\left[M_{\text {Jup }}\right]$ & $e$ & $\chi_{\text {red }}^{2}$ & r.m.s. $\left[\mathrm{ms}^{-1}\right]$ \\
\hline$\eta$ Cet $\mathrm{b}$ & $407.5 \pm 2.7$ & $2.6 \pm 0.2$ & $0.12 \pm 0.05$ & 1.013 & 15.2 \\
$\eta$ Cet $\mathrm{c}$ & $739.9 \pm 4.9$ & $3.3 \pm 0.2$ & $0.08 \pm 0.04$ & & \\
\hline \hline
\end{tabular}

around $0^{\circ}$ with very large amplitudes of $\sim \pm 180^{\circ}$ (Fig. 1 c,e). In fact as $\theta_{1}$ and $\theta_{2}$ are almost circulating, the system is very close to the separatrix.

\section{Conclusions}

We provide evidence that the $\eta$ Cet system contains two strongly interacting giant planets. The dynamical simulations show that it is very likely that the system is trapped in an anti-aligned 2:1 MMR, although a non-resonant system with near circular orbits is also possible. The $\theta_{1} \approx 180^{\circ}$ and $\theta_{2} \approx 0^{\circ} 2: 1 \mathrm{MMR}$ configuration is unusual, because it has not been seen so far in smooth migration capture (e.g., Lee 2004).

\section{References}

Chambers, J. E., 1999, MNRAS, 304, 793

Frink, S., Quirrenbach, A., Fischer, D., Röser, S., \& Schilbach, E. 2001, PASP, 113, 173

Lee, M. H. 2004, ApJ, 611, 517L 